\title{
Explicit Unsteady Navier-Stokes Solutions and their Analysis via Local Vortex
}

\section{Criteria}

Tiemo Pedergnana, ${ }^{1}$ David Oettinger, ${ }^{2}$ Gabriel P. Langlois, ${ }^{3}$ and George Haller ${ }^{4}$ a)

1) Institute of Energy Technology, ETH Zürich, Sonneggstrasse 3, 8092 Zürich, Switzerland

2) ELCA Informatik AG, Steinstrasse 21, 8003 Zürich, Switzerland

3) Division of Applied Mathematics, Brown University, Providence, RI 02912, United States

4) Institute for Mechanical Systems, ETH Zürich Leonhardstrasse 21, 8092 Zürich, Switzerland

(Dated: 13 April 2020)

We construct a class of spatially polynomial velocity fields that are exact solutions of the planar unsteady Navier-Stokes equation. These solutions can be used as simple benchmarks for testing numerical methods or verifying the feasibility of flow-feature identification principles. We use examples from the constructed solution family to illustrate deficiencies of streamlines-based feature detection and of the Okubo-Weiss criterion, which is the common two-dimensional version of the broadly used $Q_{-}^{-}, \Delta-, \lambda_{2}$ - and $\lambda_{c i}$-criteria for vortex-detection. Our planar polynomial solutions also extend directly to explicit, threedimensional unsteady Navier-Stokes solutions with a symmetry.

a)Electronic mail: georgehaller@ethz.ch 


\section{INTRODUCTION}

In this paper, we address the following question: For what time-dependent vectors $\boldsymbol{a}_{k j}(t) \in \mathbb{R}^{2}$ does the two-dimensional (2D) velocity field

$$
\mathbf{u}(\mathbf{x}, t)=\sum_{j=0}^{n} \sum_{k=0}^{m} \mathbf{a}_{k j}(t) x^{k} y^{j}
$$

solve the incompressible Navier-Stokes equation with the spatial variable $\mathbf{x}=(x, y) \in \mathbb{R}^{2}$ and the time variable $t \in \mathbb{R}$ ? Answering this question enables one to produce a large class of exact Navier-Stokes solutions for numerical benchmarking and for verifying theoretical results on simple, dynamically consistent, unsteady flow models. For linear velocity fields $\left(\mathbf{a}_{k j}(t) \equiv 0\right.$ for $k, j>1$ ), general existence conditions are detailed by Majda $\frac{1}{2}$ and Majda and Bertozzi ${ }^{2}$. A more specific form of these spatially linear solutions is given by Craik and Criminale ${ }^{3}$, who obtain that any differentiable function $\boldsymbol{a}_{00}(t)$ and any differentiable, zero-trace matrix $\mathbf{A}(t)$ generates a linear Navier-Stokes solution $\mathbf{u}(\mathbf{x}, t)$ in the form

$$
\mathbf{u}(\mathbf{x}, t)=\mathbf{a}_{00}(t)+\mathbf{A}(t) \mathbf{x},
$$

provided that $\dot{\mathbf{A}}(t)+\mathbf{A}^{2}(t)$ is symmetric. Note that all such solutions are universal (i.e., independent of the Reynolds number) because the viscous forces vanish identically on them. Reviews of exact Navier-Stokes solutions tend to omit a discussion of the Craik-Criminale solutions, although they list several specific spatially linear steady solutions for concrete physical settings (see Berker ${ }^{4}$, Wang ${ }^{5-7}$, and Drazin and Riley ${ }^{8}$ ). For solutions with at least quadratic spatial dependence, no general results of the specific form (1) have apparently been derived.

In related work, Perry and Chong 99 outline a recursive procedure for determining local Taylor expansions of solutions of the Navier-Stokes equation up to any order when specific boundary conditions are available. Bewley and Protas $\frac{10}{}$ show that near a straight boundary, the resulting Taylor coefficients can all be expressed as functions and derivatives of the skin friction and the wall pressure. The objective in these studies is, however, a recursive construction of Taylor coefficients for given boundary conditions, rather than a derivation of the general form of exact polynomial Navier-Stokes velocity fields of finite order. We also mention the work of Bajer and Moffatt ${ }^{11}$, who construct exact, steady three-dimensional (3D) Navier-Stokes flows with quadratic spatial dependence for which the flux of the velocity field through the unit sphere vanishes pointwise.

The linear part of the velocity field (1) can already have arbitrary temporal complexity, but remains spatially homogeneous by construction. The linear solution family (2) identified by Craik 
and Criminale ${ }^{3}$ cannot, therefore, produce bounded coherent flow structures. As a consequence, these linear solutions cannot yield Navier-Stokes flows with finite coherent vortices or bounded chaotic mixing zones.

Higher-order polynomial vector fields of the form (1), however, are free from these limitations, providing an endless source of unsteady and dynamically consistent examples of flow structures away from boundaries. We will construct specific examples of such flows and illustrate how the instantaneous streamlines, as well as the 2D version of the broadly used $Q$-criterion of Hunt, Wray and Moin ${ }^{12}$, the $\Delta$-criterion of ${ }^{13}$, the $\lambda_{2}$-criterion of ${ }^{14}$ and the $\lambda_{c i}$-criterion (or swirling-strength criterion) of ${ }^{15}$, fail in describing fluid particle behavior correctly in these examples. We will also point out how the two-dimensional exact solutions we construct explicitly extend to unsteady 3D Navier-Stokes solutions.

\section{UNIVERSAL NAVIER-STOKES SOLUTIONS}

We rewrite the incompressible Navier-Stokes equation (under potential body forces) for a velocity field $\mathbf{u}(\mathbf{x}, t)$ in the form

$$
\frac{\partial \mathbf{u}}{\partial t}+(\mathbf{u} \cdot \nabla) \mathbf{u}-v \Delta \mathbf{u}=\nabla\left[-\frac{p+V}{\rho}\right]
$$

where $v \geq 0$ is the kinematic viscosity, $\rho>0$ is the density, $p(\mathbf{x}, t)$ is the pressure field and $V(\mathbf{x}, t)$ is the potential of external body forces, such as gravity. We note that the left-hand side of (3) is a gradient (i.e., conservative) vector field. By classic results in potential theory, a vector field is conservative on a simply connected domain if and only if its curl is zero. For a 2D vector field, this zero-curl condition is equivalent to the requirement that the Jacobian of the vector field is zero, as already noted in the construction of linear Navier-Stokes solutions by Craik and Criminale ${ }^{3}$. On open, simply connected domains, therefore, a sufficient and necessary condition for $\mathbf{u}(\mathbf{x}, t)$ to be a Navier-Stokes solution is given by

$$
\boldsymbol{\nabla}\left[\frac{\partial \mathbf{u}}{\partial t}+(\mathbf{u} \cdot \nabla) \mathbf{u}-v \Delta \mathbf{u}\right]=\left(\nabla\left[\frac{\partial \mathbf{u}}{\partial t}+(\mathbf{u} \cdot \nabla) \mathbf{u}-v \Delta \mathbf{u}\right]\right)^{T},
$$

which no longer depends on the pressure and the external body force potential $V$. Substituting (1) into (4) and equating equal powers of $x$ and $y$ in the off-diagonal elements of the matrices on the opposite sides of the resulting equation, we obtain conditions on the unknown coefficients of the spatially polynomial velocity field $\mathbf{u}(\mathbf{x}, t)$. 
Explicit Unsteady Navier-Stokes Solutions and their Analysis via Local Vortex Criteria

By a universal solution of equation (3) we mean a solution $\mathbf{u}(\mathbf{x}, t)$ on which viscous forces identically vanish, rendering the pressure $p(\boldsymbol{x}, t)$ independent of the Reynolds number. Note that all spatially linear solutions of (3) are universal. More generally, a solution $\mathbf{u}(\mathbf{x}, t)$ of equation (3) is a universal solution of the planar Navier-Stokes equation if and only if

$$
\Delta \mathbf{u} \equiv \mathbf{0}
$$

i.e., if it is a harmonic solution. Hence, when looking for universal solutions of the form (1) that satisfy (4), we look for solutions $\mathbf{u}(\mathbf{x}, t)$ whose components are harmonic polynomials in $\mathbf{x}$ with time-dependent coefficients. Since the viscous terms in the Navier-Stokes equation vanish for harmonic flows, the universal solutions we find will also be solutions of Euler's equation. Our main result is as follows:

Theorem 1. An $n^{\text {th }}$-order, unsteady polynomial velocity field $\mathbf{u}(\mathbf{x}, t)=(u(x, y, t), v(x, y, t))$ of the spatial variable $\mathbf{x}=(x, y)$ is a universal solution of the planar, incompressible Navier-Stokes equation (3) if and only if

$$
\mathbf{u}(\mathbf{x}, t)=\mathbf{h}(t)+\frac{1}{2} \omega\left(\begin{array}{r}
-y \\
x
\end{array}\right)+\sum_{k=1}^{n}\left(\begin{array}{rr}
a_{k}(t) & b_{k}(t) \\
b_{k}(t) & -a_{k}(t)
\end{array}\right)\left(\begin{array}{c}
\operatorname{Re}(x+i y)^{k} \\
\operatorname{Im}(x+i y)^{k}
\end{array}\right)
$$

holds for some arbitrary smooth functions $\mathbf{h}: \mathbb{R} \rightarrow \mathbb{R}^{2}, a_{k}, b_{k}: \mathbb{R} \rightarrow \mathbb{R}$ and an arbitrary constant $\omega \in \mathbb{R}$, where $\omega$ coincides with the constant scalar vorticity field of $\mathbf{u}(\mathbf{x}, t)$.

Proof: To prove that a universal solution $\mathbf{u}(\mathbf{x}, t)$ must be precisely of the form given in (6), we recall from Andrews, Askey and Roy $\frac{16}{}$ that a basis of the space of $k^{\text {th }}$-order, homogeneous harmonic polynomials of two variables is given by

$$
\begin{gathered}
\left\{\operatorname{Re}\left[f_{k}(x, y)\right], \operatorname{Im}\left[f_{k}(x, y)\right]\right\}, \\
f_{k}(x, y):=(x+i y)^{k}, \quad k=0,1,2, \ldots
\end{gathered}
$$

Hence the most general form of a polynomial $\mathbf{u}(\mathbf{x}, t)$ satisfying the universality condition (5) is

$$
\mathbf{u}(\mathbf{x}, t)=\sum_{k=0}^{n}\left(\begin{array}{ll}
\alpha_{k}(t) & \beta_{k}(t) \\
\gamma_{k}(t) & \delta_{k}(t)
\end{array}\right)\left(\begin{array}{c}
\operatorname{Re}\left[f_{k}(x, y)\right] \\
\operatorname{Im}\left[f_{k}(x, y)\right]
\end{array}\right)
$$

for some smooth scalar-valued functions $\alpha_{k}(t), \beta_{k}(t), \gamma_{k}(t)$ and $\delta_{k}(t)$. By the Cauchy-Riemann equations for holomorphic complex functions, $f_{k}(x, y)$ must then satisfy

$$
\frac{\partial \operatorname{Re} f_{k}}{\partial x}=\frac{\partial \operatorname{Im} f_{k}}{\partial y}
$$


Explicit Unsteady Navier-Stokes Solutions and their Analysis via Local Vortex Criteria

$$
\frac{\partial \operatorname{Re} f_{k}}{\partial y}=-\frac{\partial \operatorname{Im} f_{k}}{\partial x}
$$

By these conditions, requiring the divergence of $\mathbf{u}(\mathbf{x}, t)$ in $(8)$ to vanish is equivalent to

$$
\begin{aligned}
\sum_{k=0}^{n}\left(\alpha_{k} \frac{\partial \operatorname{Re} f_{k}}{\partial x}+\beta_{k} \frac{\partial \operatorname{Im} f_{k}}{\partial x}+\gamma_{k} \frac{\partial \operatorname{Re} f_{k}}{\partial y}+\delta_{k} \frac{\partial \operatorname{Im} f_{k}}{\partial y}\right) & = \\
\sum_{k=0}^{n}\left(\left(\alpha_{k}+\delta_{k}\right) \frac{\partial \operatorname{Re} f_{k}}{\partial x}+\left(\gamma_{k}-\beta_{k}\right) \frac{\partial \operatorname{Re} f_{k}}{\partial y}\right) & \equiv 0 .
\end{aligned}
$$

For formula (11) to hold at order $k=0$, any constant term

$$
\mathbf{h}(t):=\left(\begin{array}{ll}
\alpha_{0}(t) & \beta_{0}(t) \\
\gamma_{0}(t) & \delta_{0}(t)
\end{array}\right)\left(\begin{array}{l}
1 \\
1
\end{array}\right)
$$

can be selected. At order $k=1$, the same formula requires

$$
\alpha_{1}(t) \equiv-\delta_{1}(t)
$$

Finally, for $k \geq 2$, formula (11) requires

$$
\alpha_{k}(t) \equiv-\delta_{k}(t), \quad \beta_{k}(t) \equiv \gamma_{k}(t), \quad k \geq 2
$$

By equations (9)-(10), the vorticity field of the $2 \mathrm{D}$ velocity field $\mathbf{u}(\mathbf{x}, t)$ is

$$
\begin{aligned}
{[\nabla \times \mathbf{u}(\mathbf{x}, t)]_{3}=\frac{\partial v}{\partial x}-\frac{\partial u}{\partial y} } & =\sum_{k=0}^{n}\left(\gamma_{k} \frac{\partial \operatorname{Re} f_{k}}{\partial x}-\alpha_{k} \frac{\partial \operatorname{Im} f_{k}}{\partial x}\right)-\left(\alpha_{k} \frac{\partial \operatorname{Re} f_{k}}{\partial y}+\beta_{k} \frac{\partial \operatorname{Im} f_{k}}{\partial y}\right) \\
& =\sum_{k=0}^{n}\left(\gamma_{k} \frac{\partial \operatorname{Re} f_{k}}{\partial x}-\beta_{k} \frac{\partial \operatorname{Im} f_{k}}{\partial y}\right)-\alpha_{k}\left(\frac{\partial \operatorname{Im} f_{k}}{\partial x}+\frac{\partial \operatorname{Re} f_{k}}{\partial y}\right) \\
& =\gamma_{1}(t)-\beta_{1}(t) .
\end{aligned}
$$

Therefore, with the notation

$$
\begin{aligned}
& a_{k}(t):=\alpha_{k}(t)=-\delta_{k}(t), \quad k \geq 1, \\
& b_{1}(t):=\frac{1}{2}\left(\beta_{1}(t)+\gamma_{1}(t)\right), \quad \omega(t):=\gamma_{1}(t)-\beta_{1}(t), \\
& b_{k}(t):=\beta_{k}(t) \equiv \gamma_{k}(t), \quad k \geq 2,
\end{aligned}
$$

and with the identity 


$$
\begin{aligned}
& \left(\begin{array}{cc}
\alpha_{1}(t) & \beta_{1}(t) \\
\gamma_{1}(t) & -\alpha_{1}(t)
\end{array}\right)\left(\begin{array}{c}
\operatorname{Re} f_{1} \\
\operatorname{Im} f_{1}
\end{array}\right)= \\
& \left(\begin{array}{cc}
\alpha_{1}(t) & \frac{1}{2}\left(\gamma_{1}(t)+\beta_{1}(t)\right) \\
\frac{1}{2}\left(\gamma_{1}(t)+\beta_{1}(t)\right) & -\alpha_{1}(t)
\end{array}\right)\left(\begin{array}{c}
\operatorname{Re} f_{1} \\
\operatorname{Im} f_{1}
\end{array}\right)+ \\
& \left(\begin{array}{cc}
0 & \frac{1}{2}\left(\gamma_{1}(t)+\beta_{1}(t)\right) \\
\frac{1}{2}\left(\gamma_{1}(t)+\beta_{1}(t)\right) & 0
\end{array}\right)\left(\begin{array}{c}
\operatorname{Re} f_{1} \\
\operatorname{Im} f_{1}
\end{array}\right) \\
& =\left(\begin{array}{cc}
\alpha_{1}(t) & \left.b_{1}(t)\right) \\
\left.b_{1}(t)\right) & -\alpha_{1}(t)
\end{array}\right)\left(\begin{array}{c}
\operatorname{Re} f_{1} \\
\operatorname{Im} f_{1}
\end{array}\right)+\left(\begin{array}{cc}
0 & -\frac{1}{2} \omega(t) \\
\frac{1}{2} \omega(t) & 0
\end{array}\right)\left(\begin{array}{l}
x \\
y
\end{array}\right),
\end{aligned}
$$

formulas (8)-(13) give

$$
\mathbf{u}(\mathbf{x}, t)=\mathbf{h}(t)+\frac{1}{2} \omega(t)\left(\begin{array}{r}
-y \\
x
\end{array}\right)+\sum_{k=1}^{n}\left(\begin{array}{rr}
a_{k}(t) & b_{k}(t) \\
b_{k}(t) & -a_{k}(t)
\end{array}\right)\left(\begin{array}{c}
\operatorname{Re} f_{k} \\
\operatorname{Im} f_{k}
\end{array}\right),
$$

with $\omega(t)$ denoting the spatially constant (but at this point time-dependent) scalar vorticity field of (14). Since $\mathbf{u}(\mathbf{x}, t)$ is harmonic and incompressible, the symmetry condition (4) is satisfied by the velocity field (14) if and only if the $2 \times 2$ matrix

$$
\nabla \frac{\partial \mathbf{u}}{\partial t}+\nabla[(\mathbf{u} \cdot \nabla) \mathbf{u}]
$$

is symmetric. To verify the symmetry of this matrix using the notation $\mathbf{u}=(u, v)$, first note that the skew-symmetric parts of the two summands in (15) are given by

$$
\begin{aligned}
\text { skew }\left[\nabla \frac{\partial \mathbf{u}}{\partial t}\right] & =\frac{1}{2}\left(\nabla \frac{\partial u}{\partial t}-\left[\nabla \frac{\partial u}{\partial t}\right]^{T}\right)=\frac{1}{2}\left(\begin{array}{rr}
0 & -\dot{\omega}(t) \\
\dot{\omega}(t) & 0
\end{array}\right), \\
\operatorname{skew}[\nabla[(\mathbf{u} \cdot \nabla) \mathbf{u}]] & =\operatorname{skew}\left(\begin{array}{cr}
0 & u_{x y} u+u_{y y} v+\left(u_{x}+v_{y}\right) u_{y} \\
v_{x x} u+v_{x y} v+\left(u_{x}+v_{y}\right) v_{x} & 0
\end{array}\right)=\mathbf{0},
\end{aligned}
$$

where we have used the incompressibility condition $u_{x}+v_{y}=0$, as well as the spatial independence of the scalar vorticity $\omega(t)$ of $\mathbf{u}$, which implies $\partial_{x} \omega=v_{x x}-u_{x y} \equiv 0$ and $\partial_{y} \omega=v_{x y}-u_{y y} \equiv 0$. Formulas (15)-(17) then imply that for the matrix (15) to be symmetric, we must have $\dot{\omega}(t) \equiv 0$, i.e., $\omega(t)=\omega=$ const. must hold in (14). This completes the proof of the theorem.

For the universal solutions derived in this section, the pressure field $p(\mathbf{x}, t)$ can be obtained by substituting the solutions into (3), integrating the left-hand side of the resulting equation, multiplying the result by $-\rho$ and subtracting the potential $V(\mathbf{x}, t)$. 
The two-dimensional Navier-Stokes solution family (6) immediately generates three-dimensional, incompressible Navier-Stokes solutions as well. The planar components of these solutions are just given by (6), while their vertical component, $w(x, y, t)$, satisfies the scalar advection-diffusion equation

$$
\partial_{t} w+\nabla w \cdot \mathbf{u}=v \Delta w
$$

as shown, e.g., by Majda and Bertozzi ${ }^{2}$. We therefore obtain the following result.

Proposition 2. Any polynomial solution (14) of the planar Navier-Stokes equation generates a family of exact solutions $(\mathbf{u}(\mathbf{x}, t), w(\mathbf{x}, t))$ for the three-dimensional version of the Navier-Stokes equation (3), where $w(\mathbf{x}, t)$ is an arbitrary solution of the advection-diffusion eq. (18). In particular,

$$
\mathbf{v}(\mathbf{x}, t)=\left(\begin{array}{c}
\mathbf{u}(\mathbf{x}, t) \\
w(\mathbf{x}, t)
\end{array}\right)=\left(\begin{array}{c}
\mathbf{h}(t) \\
w_{0}
\end{array}\right)+\frac{1}{2} \omega(t)\left(\begin{array}{c}
-y \\
x \\
0
\end{array}\right)+\left(\sum_{k=1}^{n}\left(\begin{array}{cc}
a_{k}(t) & b_{k}(t) \\
b_{k}(t) & -a_{k}(t)
\end{array}\right)\left(\begin{array}{c}
\operatorname{Re} f_{k} \\
\operatorname{Im} f_{k}
\end{array}\right)\right.
$$

is an exact, unsteady polynomial solution of the 3D Navier-Stokes equation for any choice of the constant vertical velocity $w_{0} \in \mathbb{R}$.

\section{VORTEX IDENTIFICATION METHODS}

For our later analysis of universal solution examples, we now briefly review the most broadly used pointwise structure identification schemes in unsteady flows. For 2D flows, the Okubo-Weiss criterion $\left(\mathrm{Okubo}^{17}\right.$, Weiss $\left.{ }^{18}\right)$ postulates that the nature of fluid particle motion in a flow is governed by the eigenvalue configuration of the velocity gradient $\nabla \mathbf{u}(\mathbf{x}, t)$. For $2 \mathrm{D}$ incompressible flows, this eigenvalue configuration is uniquely characterized by the scalar field

$$
O W(\mathbf{x}, t)=-\operatorname{det}[\nabla \mathbf{u}(\mathbf{x}, t)]
$$

The Okubo-Weiss criterion postulates that in elliptic (or vortical) regions, the velocity gradient $\nabla \mathbf{u}(\mathbf{x}, t)$ has purely imaginary eigenvalues, or equivalently, $O W(\mathbf{x}, t)<0$ holds. Similarly, the criterion postulates that hyperbolic (or stretching) regions are characterized by $O W(\mathbf{x}, t)>0$.

As formula (19) shows, any universal 2D Navier-Stokes solutions generates a 3D NavierStokes solution family $\mathbf{v}=\left(\mathbf{u}, w_{0}\right)$ with an arbitrary, constant vertical velocity component $w_{0}$. 
This extension enables the application of 3D local vortex-identification criteria to our universal solutions. Such criteria generally involve the spin tensor $\Omega=\frac{1}{2}\left[\nabla \mathbf{v}-(\nabla \mathbf{v})^{T}\right]$ and the rate-ofstrain tensor $\mathbf{S}=\frac{1}{2}\left[\nabla \mathbf{v}+(\nabla \mathbf{v})^{T}\right]$, the antisymmetric and symmetric parts of $\nabla \mathbf{v}$, respectively.

While Epps ${ }^{19}$ provides a comprehensive review of such criteria, we will here focus on the three most broadly used ones.

We also mention the work of Rousseaux et al. ${ }^{20}$, who utilize the hydrodynamic Lamb vector (cf. Belevich $\stackrel{21}{ }$, Marmanis $\frac{22}{2}$ and Sridhar $\stackrel{23}{ }$ ) in vortex detection.

The first of the three local vortex criteria we discuss here, the $Q$-criterion of Hunt, Wray and Moin ${ }^{12}$, postulates that in a vortical region, the Euclidean matrix norm of $\Omega$ dominates that of $\mathbf{S}$, rendering the scalar field

$$
Q(\mathbf{x}, t)=\frac{1}{2}\left(\|\Omega\|^{2}-\|\mathbf{S}\|^{2}\right)
$$

positive. One can verify by direct calculation that $Q(\mathbf{x}, t) \equiv O W(\mathbf{x}, t)$ holds for the $3 \mathrm{D}$ extension (19) of our universal solutions (and, in general, for any two-dimensional flow), rendering the $Q$ criterion equivalent to the Okubo-Weiss criterion for these flows.

Second, the $\Delta$-criterion of Chong et al. ${ }^{13}$ seeks vortices in $3 \mathrm{D}$ flow as domains where the velocity gradient $\nabla \mathbf{v}(\mathbf{x}, t)$ admits eigenvalues with nonzero imaginary parts. In the extended universal solutions (19), zero is always an eigenvalue for $\nabla \mathbf{v}$ and hence, by incompressibility, the remaining two eigenvalues of $\nabla \mathbf{v}$ are complex precisely when they are purely imaginary. This happens precisely when $Q(\mathbf{x}, t) \equiv O W(\mathbf{x}, t)>0$ holds, and hence the $\Delta$-criterion also coincides with the Okubo-Weiss criterion on the universal solutions we have constructed.

Third, the $\lambda_{2}$-criterion of Jeong and Hussein ${ }^{14}$ identifies vortices as the collection of points where the pressure has a local minimum within an appropriately chosen two-dimensional plane. Under various further assumptions, this principle is equivalent to the requirement that the intermediate eigenvalue, $\lambda_{2}\left(\mathbf{S}^{2}+\mathbf{W}^{2}\right)$, of the symmetric tensor $\mathbf{S}^{2}+\mathbf{W}^{2}$ must satisfy.

$$
\lambda_{2}\left(\mathbf{S}^{2}+\mathbf{W}^{2}\right)<0
$$

For the extended universal solutions (19), we obtain from a direct calculation that

$$
\lambda_{2}\left(\mathbf{S}^{2}+\mathbf{W}^{2}\right)=S_{11}^{2}+S_{12}^{2}-W_{12}^{2}=-Q,
$$

the $\lambda_{2}$-criterion also agrees with Okubo-Weiss criterion for these flows.

Finally, the $\lambda_{c i}$-criterion (or swirling-strength criterion) of Chakraborthy et al. $\frac{15}{}$ follows the logic of the $\Delta$-criterion and asserts that local material swirling occurs at points where $\nabla \mathbf{v}$ has a 
pair of complex eigenvalues $\lambda_{c r}+i \lambda_{c i}$ and a real eigenvalue $\lambda_{r}$. To ensure tight enough spiraling (orbital compactness) typical for a vortex, the $\lambda_{c i}$-criterion asserts the requirement

$$
\lambda_{c i} \geq \varepsilon, \quad \lambda_{c r} / \lambda_{c i} \leq \delta
$$

for some small, constant thresholds $\varepsilon, \delta>0$. Again, for the extended universal flows (19), we can only have $\lambda_{c i}=0$ and on any compact domain with $\lambda_{c i}>0$ we can select an $\varepsilon>0$ such that the criterion (22) is satisfies. Once again, therefore, the $\lambda_{c i}$-criterion agrees with the Okubo-Weiss criterion for the the extended universal flows (19).

All these vortex criteria seek to capture vortex-type fluid particle behavior in a heuristic fashion, i.e., hope to achieve conclusions about the trajectories generated by the velocity field $\mathbf{v}(\mathbf{x}, t)$ from instantaneous snapshots of $\mathbf{v}(\mathbf{x}, t)$. Some of these criteria have been shown to give reasonable results on simple steady flows. Such steady examples have prompted the broad use of these criteria in analyzing general unsteady flow data for which no ground truth is available. This practice can immediately be questioned based on first principles, given that all these criteria are framedependent (see Haller ${ }^{24,25}$ ), yet truly unsteady flows have no distinguished frames (cf. Lugt ${ }^{26}$ ). Another questionable practice has been to simply replace the original versions of vortex identification criteria with plots of heuristically chosen level surfaces of the quantities arising in these criteria. Such level surfaces are generally highly sensitive to the choice of the constant value that defines them, yet these constants are routinely chosen to match intuition or provide visually pleasing results.

The exact, unsteady Navier-Stokes solutions we have constructed here give an opportunity to test the vortex identification methods above on nontrivial, unsteady Navier-Stokes solutions in which a ground truth can be reliably established via Poincaré maps. As we will see below, such a systematic analysis reveals major inconsistencies for all the vortex criteria recalled above. As we have seen, it will be enough to point out these inconsistencies on the 2D universal solutions (6) for the Okubo-Weiss criterion. The same inconsistencies arise then automatically in the analysis of the extended 3D Navier-Stokes solution (19) via the $Q_{-}^{-}, \Delta^{-}, \lambda_{2^{-}}$and $\lambda_{c i}$-criteria.

\section{EXAMPLES}

We now give examples of dynamically consistent flow fields covered by the general formula (6). With these exact solutions, we illustrate that using the instantaneous streamlines for material 
vortex detection (see, e.g., Sadajoen and $\operatorname{Post}^{27}$ and Robinson ${ }^{28}$ ) gives inconsistent results. With the same solutions, we also illustrate how the Okubo-Weiss criterion described in the previous section fails to identify the true nature of unsteady fluid particle motion.

Example 1. Haller ${ }^{24,25}$ proposed the velocity field

$$
\mathbf{u}(\mathbf{x}, t)=\left(\begin{array}{cc}
\sin 4 t & \cos 4 t+2 \\
\cos 4 t-2 & -\sin 4 t
\end{array}\right) \mathbf{x}
$$

as a purely kinematic benchmark example for testing vortex criteria. By inspection of (6), we find that (24) solves the Navier-Stokes equation with $\mathbf{h}(t) \equiv \mathbf{0}, a_{1}(t)=\sin 4 t, b_{1}(t)=\cos 4 t, \omega=-4$, and $a_{k}=b_{k} \equiv 0$ for $k \geq 2$. More generally, formula (6) shows that the linear unsteady velocity field

$$
\dot{\mathbf{x}}=\mathbf{u}(\mathbf{x}, t)=\left(\begin{array}{cc}
-\sin C t & \cos C t-\frac{\omega}{2} \\
\cos C t+\frac{\omega}{2} & \sin C t
\end{array}\right) \mathbf{x}
$$

solves the 2D Navier-Stokes equation for any constants $\omega$ and $C$, and any smooth function $\mathbf{h}(t)$. We set $\mathbf{h}(t) \equiv \mathbf{0}$ for simplicity and pass to a rotating $\mathbf{y}$-coordinate frame via the transformation

$$
\mathbf{x}=\mathbf{M}(t) \mathbf{y}, \quad \mathbf{M}(t)=\left(\begin{array}{cc}
\cos \frac{C}{2} t & \sin \frac{C}{2} t \\
-\sin \frac{C}{2} t & \cos \frac{C}{2} t
\end{array}\right) .
$$

In these new coordinates, (25) becomes

$$
\begin{aligned}
\dot{\mathbf{x}} & =\left(\begin{array}{cc}
-\sin C t & \cos C t-\frac{\omega}{2} \\
\cos C t+\frac{\omega}{2} & \sin C t
\end{array}\right) \mathbf{x} \\
& =\left(\begin{array}{cc}
-\sin C t & \cos C t-\frac{\omega}{2} \\
\cos C t+\frac{\omega}{2} & \sin C t
\end{array}\right) \mathbf{M}^{-1} \mathbf{y} \\
& =\left(\begin{array}{cc}
-\left(1+\frac{\omega}{2}\right) \sin \frac{C}{2} t & \left(1-\frac{\omega}{2}\right) \cos \frac{C}{2} t \\
\left(1+\frac{\omega}{2}\right) \cos \frac{C}{2} t & \left(1-\frac{\omega}{2}\right) \sin \frac{C}{2} t
\end{array}\right) \mathbf{y} .
\end{aligned}
$$

At the same time, differentiating the coordinate change (26) with respect to time gives $\dot{\mathbf{x}}=$ $\dot{\mathbf{M y}}+\mathbf{M y}$, which, combined with (28) gives the velocity field in the $\mathbf{y}$-frame as

$$
\dot{\mathbf{y}}=\tilde{\mathbf{u}}(\mathbf{y})=\left(\begin{array}{cc}
0 & 1+\frac{1}{2}(C-\omega) \\
1-\frac{1}{2}(C-\omega) & 0
\end{array}\right) \mathbf{y} .
$$

This transformed velocity field is steady, defining an exactly solvable autonomous linear system of differential equations for particle motions. The nature of its solutions depends on the eigenvalues 
(a)

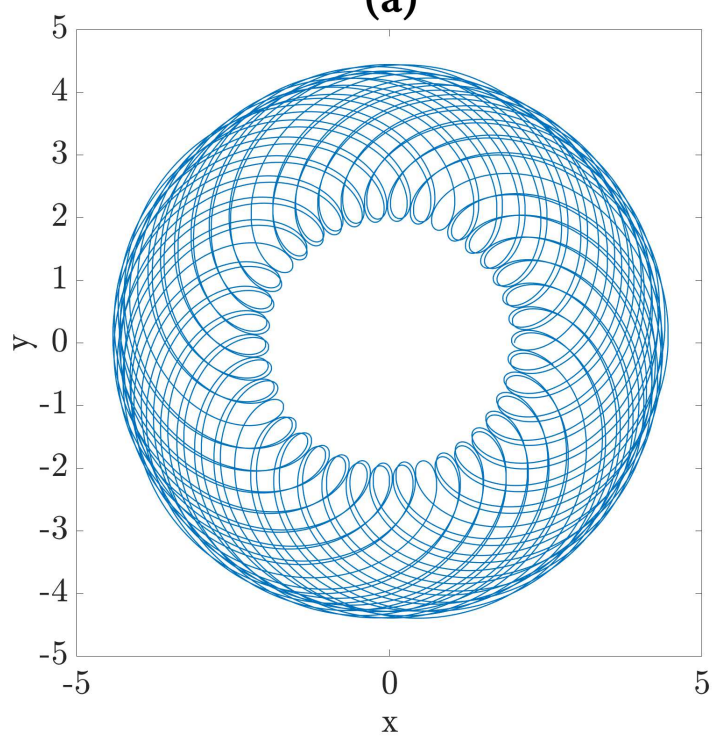

(b)

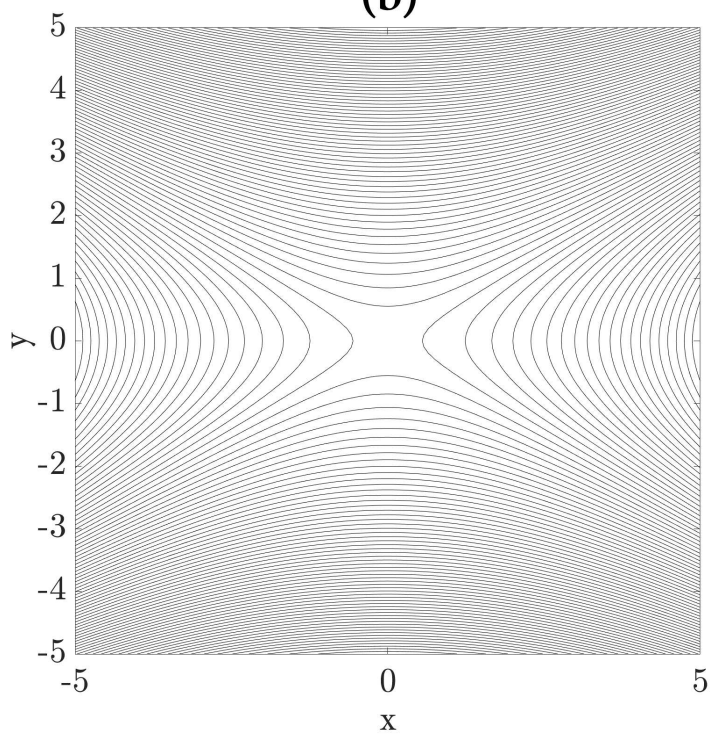

FIG. 1. (a) A typical fluid particle trajectory generated by the linear unsteady velocity field (30) for the time interval $\left[t_{0}, t_{1}\right]=[0,200]$ for the initial condition $\boldsymbol{x}_{0}=(2,0)$. This flow would appear as an unbounded vortex in any flow-visualization experiment involving dye or particles. (b) The instantaneous streamlines of the same velocity field, shown here for $t=0$, suggest a saddle point at the origin for all times (streamlines at other times look similar).

$\lambda_{1,2}= \pm \sqrt{1-\frac{1}{4}(\omega-C)^{2}}$ of the coefficient matrix in (26). Specifically, for $|\omega-C|<2$, we have a saddle-type flow with typical solutions growing exponentially, while for $|\omega-C|>2$, we have a center-type flow in which all trajectories perform periodic motion.

Mapped back into the original frame via the time-periodic transformation (26), the center-type trajectories become quasiperiodic. Figure 1(a) shows such a quasiperiodic particle trajectory and Fig. 1(b) instantaneous streamlines of the velocity field (6) with $\mathbf{h}(t) \equiv \mathbf{0}, a_{1}(t)=\sin 4 t, b_{1}(t)=$ $\cos 4 t$, and $\omega=-1$ and $a_{k}=b_{k} \equiv 0$ for $k \geq 2$. These parameter values yield the velocity field

$$
\mathbf{u}(\mathbf{x}, t)=\left(\begin{array}{cc}
\sin 4 t & \cos 4 t+\frac{1}{2} \\
\cos 4 t-\frac{1}{2} & -\sin 4 t
\end{array}\right) \mathbf{x},
$$

which satisfies $|\omega-C|>2$. This flow would, therefore, appear as an unbounded vortex in any flow visualization experiment involving dye or particles, yet its instantaneous streamlines suggest a saddle point at the origin for all times. Similarly, the Okubo-Weiss criterion incorrectly 
pronounces the entire plane hyperbolic for the flow (30) for all times. Indeed, formula (20) gives

$$
O W \equiv 1-\frac{\omega^{2}}{4}=\frac{7}{16}>0
$$

Example 2. By the general formula (6), a simple quadratic extension of the linear velocity field (24) is given by the universal Navier-Stokes solution

$$
\mathbf{u}(\mathbf{x}, t)=\left(\begin{array}{cc}
\sin 4 t & \cos 4 t+2 \\
\cos 4 t-2 & -\sin 4 t
\end{array}\right) \mathbf{x}+\alpha(t)\left(\begin{array}{c}
x^{2}-y^{2} \\
-2 x y
\end{array}\right)
$$

where we have chosen $a_{2}(t) \equiv \alpha(t)$ and $b_{2}(t) \equiv 0$ in the quadratic terms of (6) , and set $\mathbf{h}(t), a_{k}(t)$ and $b_{k}$ for $k>2$, as in Example 1. Setting $\alpha(t) \equiv-0.1$ for simplicity, we find that the instantaneous streamlines now suggest a bounded spinning vortex enclosed by connections between two stagnation points. The Okubo-Weiss criterion also suggests a coherent vortex surrounding the origin at all times, as $O W<0$ holds on a yellow domain shown containing the origin, as shown in Fig. 2 (a). In reality, however, the origin is a saddle-type Lagrangian trajectory with transversely intersecting stable and unstable manifolds.

Shown in Fig. 2(b) for the Poincare map of the flow, the resulting homoclinic tangle creates intense chaotic mixing. This mixing process rapidly removes all but a measure zero set of initial conditions from the Okubo-Weiss vortical region. Therefore, the Navier-Stokes solution (32) with $\alpha(t) \equiv-0.1$ provides a clear false positive for coherent material vortex detection based on streamlines and on the Okubo-Weiss criterion.

Example 3. Building on the discussion of the stability of the $x=0$ fixed point of equation (25), we now consider another specific Navier-Stokes velocity field of the form

$$
\mathbf{u}(\mathbf{x}, t)=\left(\begin{array}{cc}
\sin 4 t & \cos 4 t+\frac{1}{2} \\
\cos 4 t-\frac{1}{2} & -\sin 4 t
\end{array}\right) \mathbf{x}+\alpha(t)\left(\begin{array}{c}
x^{2}-y^{2} \\
-2 x y
\end{array}\right),
$$

from the universal solution family (6). In the notation used for equation (25), we now have $\omega=$ -1 and $C=4$, which gives $|C-\omega|>2$. Therefore, as discussed in Example 1, the origin of (33) is a center-type fixed point under linearization for the Lagrangian particle motion. At the same time, both the instantaneous streamlines in Fig. 3(a) and the Okubo-Weiss criterion suggest saddle-type (hyperbolic) behavior for the linearized flow, given that $O W>0$ holds on the whole plane. By the Kolmogorov-Arnold-Moser (KAM) theorem ${ }^{29}$, however, setting the small parameter $\alpha(t) \equiv$ -0.015 in (33) is expected to preserve the elliptic (vortical) nature of the Lagrangian particle 
(a)

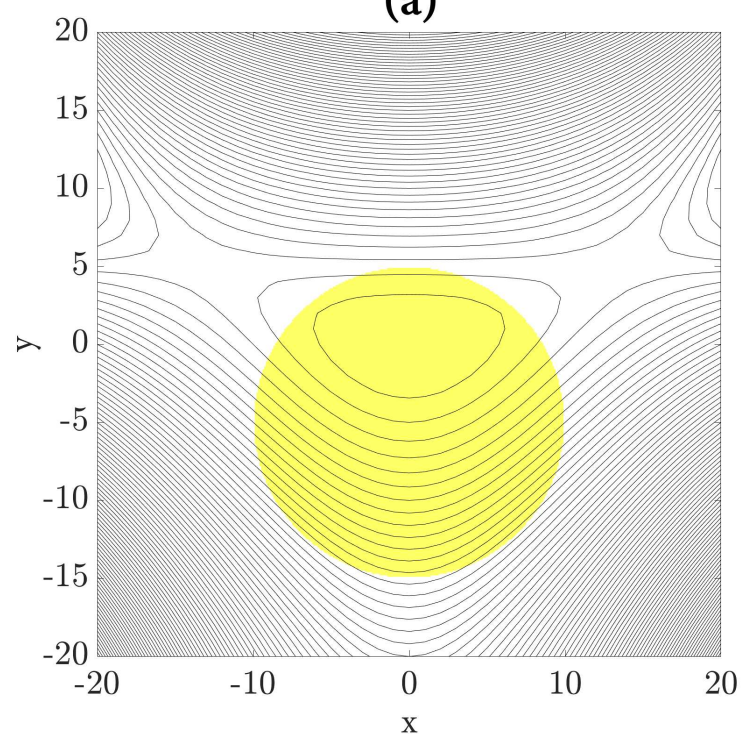

(b)

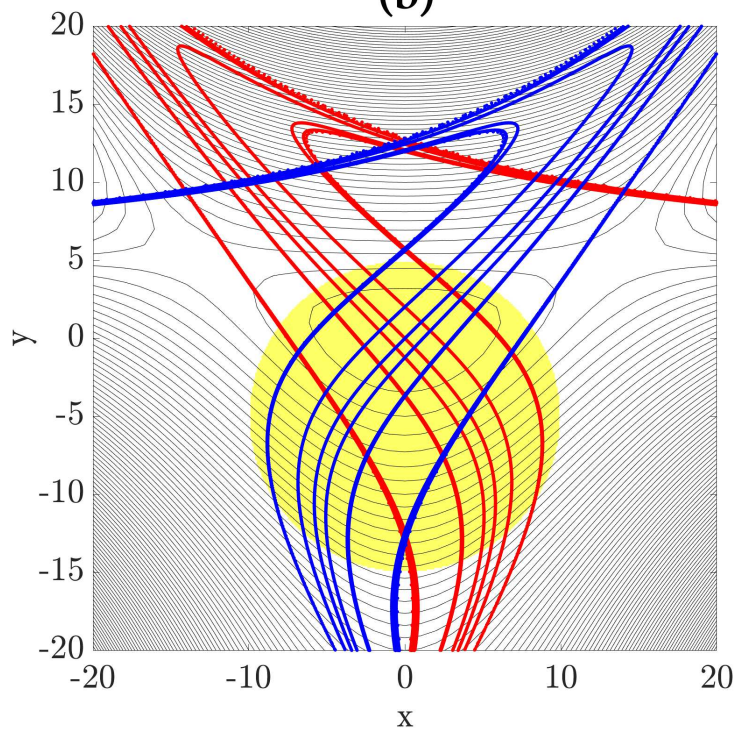

FIG. 2. (a) Instantaneous streamlines and Okubo-Weiss elliptic region (yellow) for the universal NavierStokes solution (32) with $\alpha(t) \equiv-0.1$ at time $t=0$. Other time slices are similar. (b) Stable (blue) and unstable (red) manifolds of the fixed point of the Poincaré map (based at $t=0$ with period $T=\frac{\pi}{2}$ ) for the Lagrangian particle motions under the same velocity field, superimposed on the structures shown in (a).

motion in the quadratic velocity field (33). Indeed, most quasiperiodic motions of the linearized system survive with the exception of resonance islands, as indicated by the KAM curves shown in blue in Fig. 3(b). Therefore, the Navier-Stokes solution (33) with $\alpha(t) \equiv-0.015$ provides a false negative for coherent material vortex detection based on streamlines or the Okubo-Weiss criterion. Note that the KAM curves shown in some of the examples in this section were obtained by launching fluid particles from a uniformly spaced grid over the domain shown, advecting them over the time span $\left[\begin{array}{ll}0 & 2 \pi\end{array}\right]$, and plotting the advected positions of the fluid particles at each time step.

Example 4. By the general formula (6), a cubic extension of (24) is given by the universal NavierStokes solution

$$
\mathbf{u}(\mathbf{x}, t)=\left(\begin{array}{cc}
\sin 4 t & \cos 4 t+2 \\
\cos 4 t-2 & -\sin 4 t
\end{array}\right) \mathbf{x}+\alpha(t)\left(\begin{array}{c}
x\left(x^{2}-3 y^{2}\right) \\
-y\left(3 x^{2}-y^{2}\right)
\end{array}\right),
$$

where we have chosen $a_{2}(t) \equiv 0, b_{2}(t) \equiv 0, a_{3}(t) \equiv \alpha(t)$ and $b_{3}(t) \equiv 0$ in the quadratic and cubic 
(a)

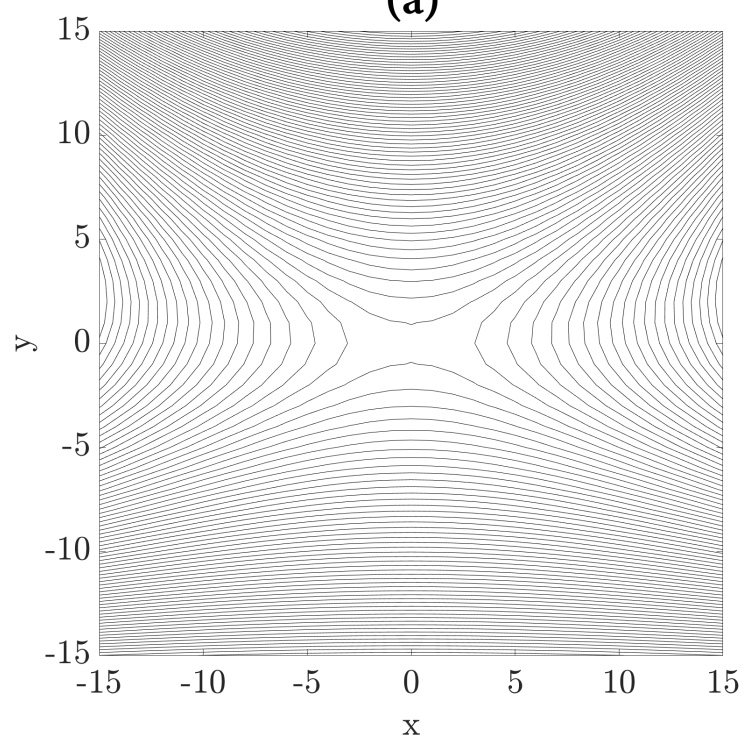

(b)

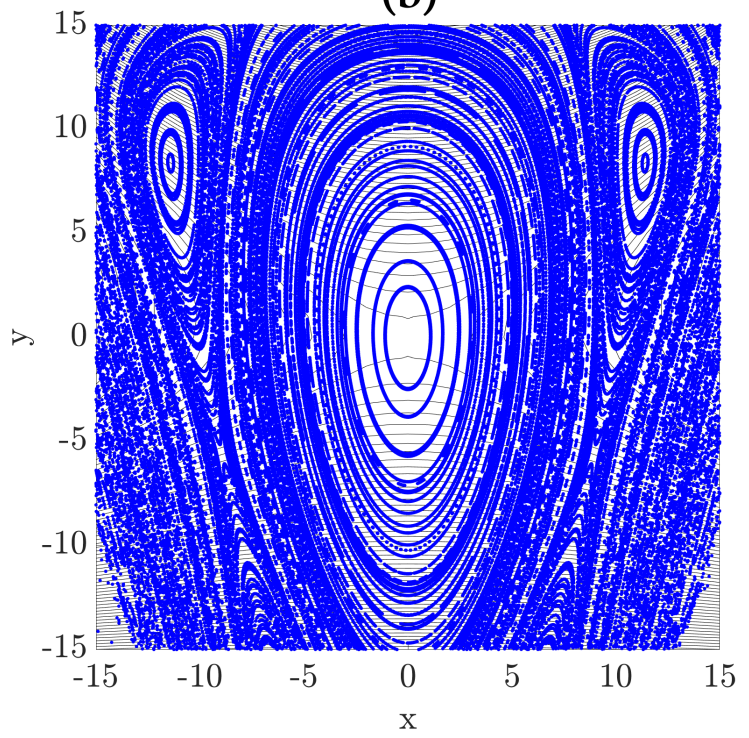

FIG. 3. (a) Instantaneous streamlines for the universal Navier-Stokes solution (33) with $\alpha(t) \equiv-0.015$ at time $t=0$. Other time slices are similar. (b) KAM curves (blue) of the same velocity field, superimposed on the streamlines shown in (a).

terms of (6), respectively, and set $\mathbf{h}(t), \omega, a_{k}(t)$ and $b_{k}(t)$ for $k>3$ as in Example 2. We also set $\alpha(t) \equiv 0.005$. The instantaneous streamlines, shown in Fig. 4(a) for $t=0$, suggest a bounded spinning vortex around the origin surrounded by two saddle-type structures. Similarly, the OkuboWeiss criterion, visualized by the yellow domain $(O W<0)$ in Fig.4(a), suggests a coherent vortex surrounding the origin at all times.

The actual Lagrangian dynamics, however, is again strikingly different: Shown in Fig. 4(b), the Poincare map of the flow shows that the origin is a saddle-type Lagrangian trajectory with transversely intersecting stable (blue) and unstable (red) manifolds, which lead to chaotic mixing near the origin. Therefore, the Navier-Stokes solution (34) with $\alpha(t) \equiv 0.005$ provides, similarly to Example 2, a false positive for coherent material vortex detection based on streamlines and on the Okubo-Weiss criterion.

Example 5. We now consider another universal Navier-Stokes solution of the form

$$
\mathbf{u}(\mathbf{x}, t)=\left(\begin{array}{cc}
\sin 4 t & \cos 4 t+\frac{1}{2} \\
\cos 4 t-\frac{1}{2} & -\sin 4 t
\end{array}\right) \mathbf{x}+\alpha(t)\left(\begin{array}{c}
x\left(x^{2}-3 y^{2}\right) \\
-y\left(3 x^{2}-y^{2}\right)
\end{array}\right)
$$


(a)

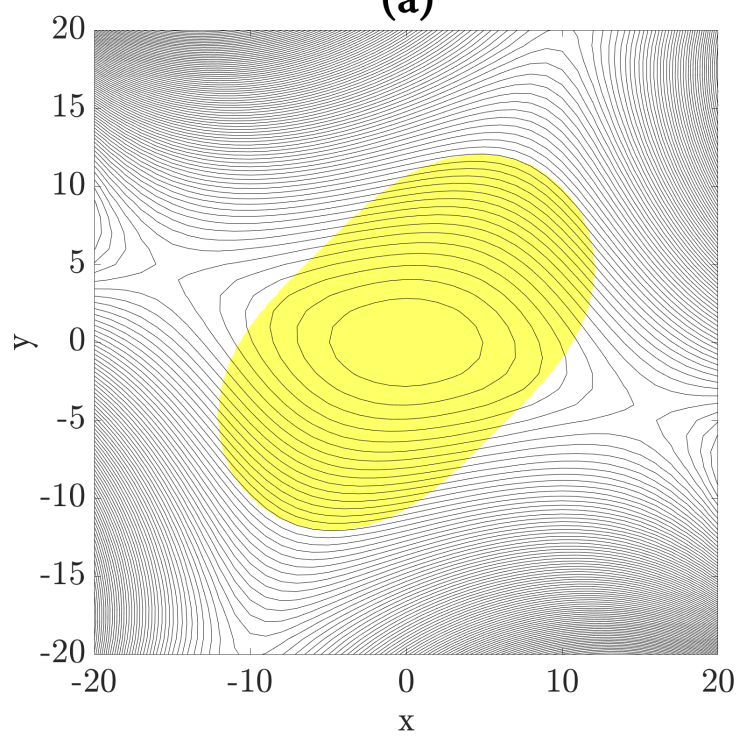

(b)

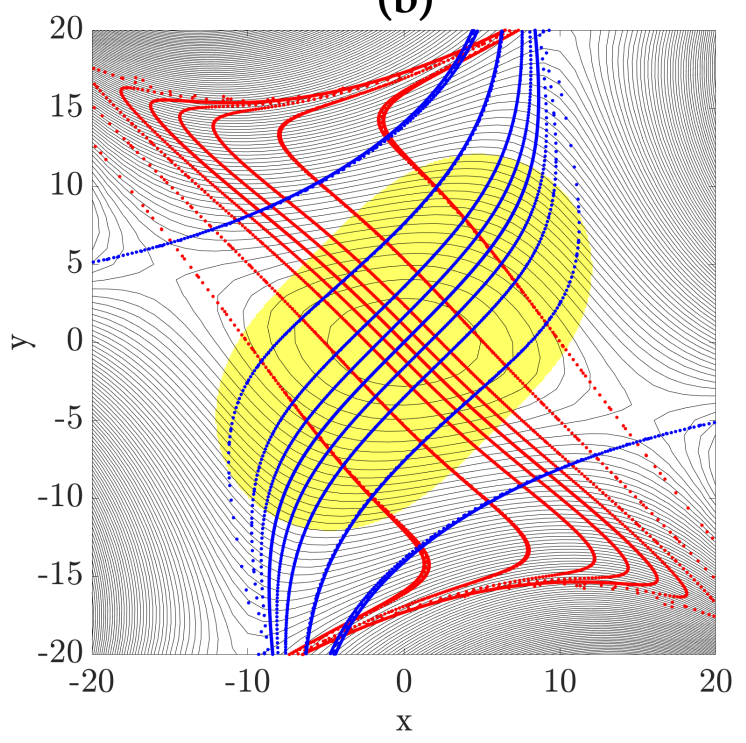

FIG. 4. (a) Instantaneous streamlines and Okubo-Weiss elliptic region (yellow) for the universal NavierStokes solution (34) with $\alpha(t) \equiv 0.005$ at time $t=0$. Other time slices are similar. (b) Stable (blue) and unstable (red) manifolds of the fixed point of the Poincare map (based at $t=0$ with period $T=\frac{\pi}{2}$ ) for the Lagrangian particle motions under the same velocity field, superimposed on the structures shown in (a).

where we have chosen $a_{2}(t) \equiv 0, b_{2}(t) \equiv 0, a_{3}(t) \equiv \alpha(t)$ and $b_{3}(t) \equiv 0$ in the quadratic and cubic terms of (6), respectively, and selected $\mathbf{h}(t), \omega, a_{k}(t)$ and $b_{k}(t)$, for $k=2$ and $k>3$ as in Example 1. As discussed in Example 3, for the choice of a small parameter $\alpha(t) \equiv 0.005$, we expect, by the KAM theorem ${ }^{29}$, that most quasiperiodic motions linearized system survive around the origin. Contrary to this, the instantaneous streamline picture, shown in Fig. 5(a) for $t=0$, suggests a stagnation point at the origin. The Okubo-Weiss criterion, visualized by the yellow domains $(O W<0)$ in Fig. 5(a), predicts two coherent vortices away from the origin. Fig. 5(b) shows in blue KAM curves of the velocity field (35), revealing a bounded material coherent vortex around the origin. The rest of the particles, which are not captured by the material vortex, escape to infinity. Hence the Okubo-Weiss criterion provides one false negative and two false positives for vortex identification in the velocity field (35).

Example 6. A further extension of the velocity field (35) is given by the universal Navier-Stokes solution 
(a)

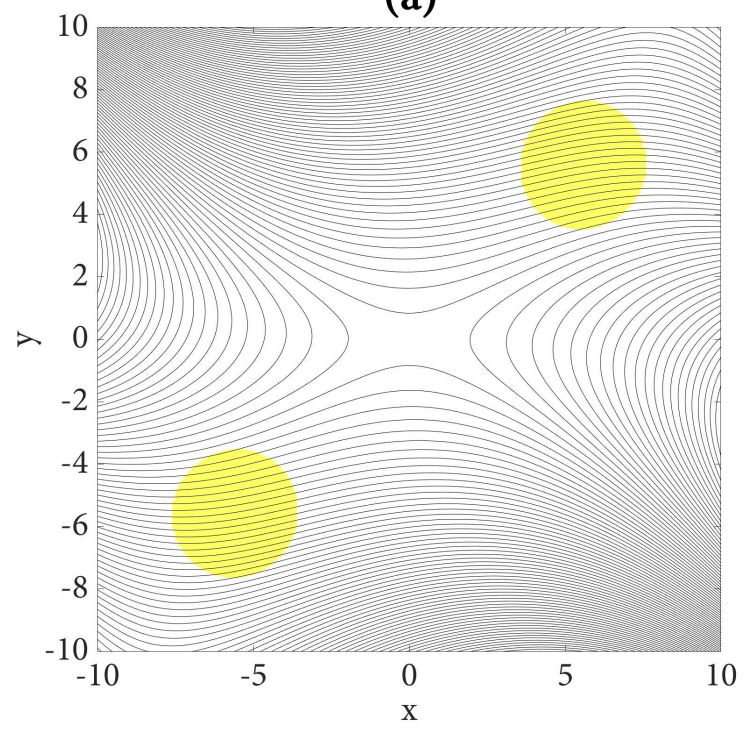

(b)

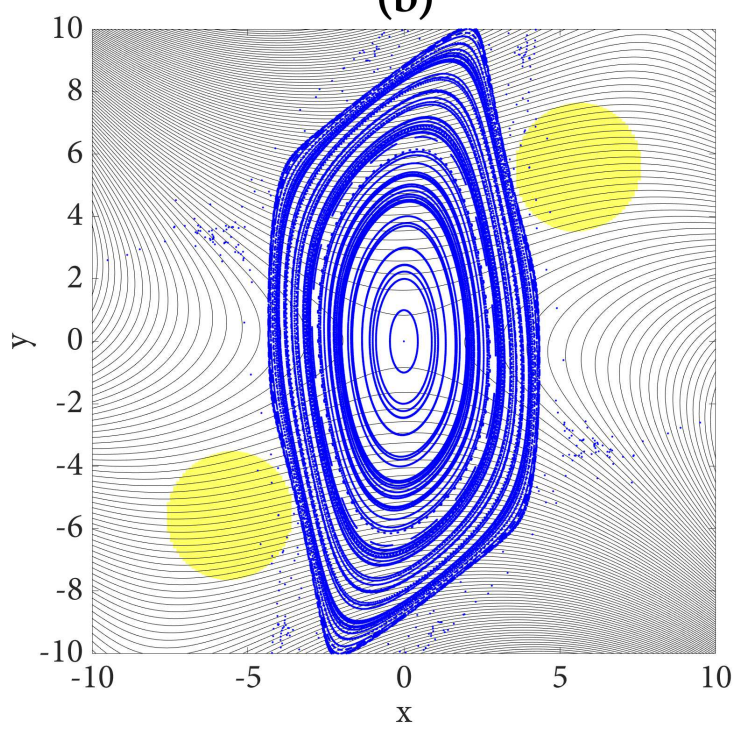

FIG. 5. (a) Instantaneous streamlines and Okubo-Weiss elliptic region (yellow) for the universal NavierStokes solution (35) with $\alpha(t) \equiv 0.005$ at time $t=0$. Other time slices are similar. (b) KAM curves (blue) of the same velocity field.

$$
\mathbf{u}(\mathbf{x}, t)=\left(\begin{array}{cc}
\sin 10 t & \cos 10 t+2 \\
\cos 10 t-2 & -\sin 10 t
\end{array}\right) \mathbf{x}+\alpha(t)\left(\begin{array}{c}
x\left(x^{2}-3 y^{2}\right) \\
-y\left(3 x^{2}-y^{2}\right)
\end{array}\right)
$$

where we have chosen $a_{2}(t) \equiv 0, b_{2}(t) \equiv 0, a_{3}(t) \equiv \alpha(t)$ and $b_{3}(t) \equiv 0$ in the quadratic and cubic terms of (6), respectively, and set $\mathbf{h}(t), \alpha(t), \omega, a_{k}(t)$ and $b_{k}(t)$ for $k>3$ as in Example 4. This solution is a nonlinear extension of the general linear velocity field (25), with $\omega=-2$ and $C=10$. Therefore, as for the solution (33), $|C-\omega|>2$. Hence the origin of (36) is a center-type fixed point of the linearized system. By the KAM theorem ${ }^{29}$, adding a nonlinear term multiplied by the small parameter $\alpha(t) \equiv 0.005$ in (36), the Lagrangian particle motion in the cubic velocity field (36) is expected to remain elliptical (vortical) around the origin. The instantaneous streamlines of (36), shown in Fig. 6(a) for $t=0$, suggest a coherent vortex around the origin. The OkuboWeiss criterion, visualized in Fig. 6(a) for the initial time $t=0$ by the yellow domain $(O W>0)$, also suggests a coherent vortex near the origin. Shown in blue in Fig. 6(b) are KAM curves of the flow, revealing indeed a bounded coherent vortex around the origin. The rest of the particles, which are not captured by the material vortices around the origin, escape to infinity. Hence for the 
(a)

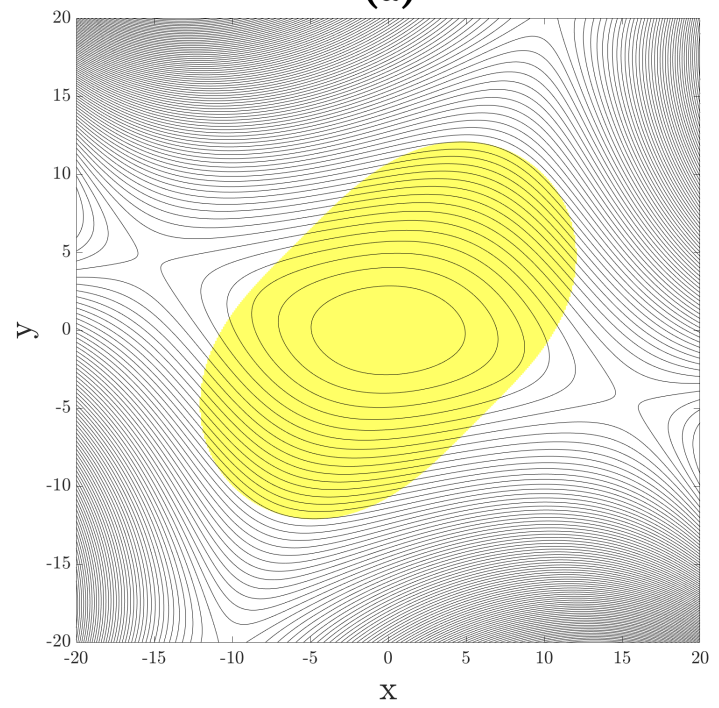

(b)

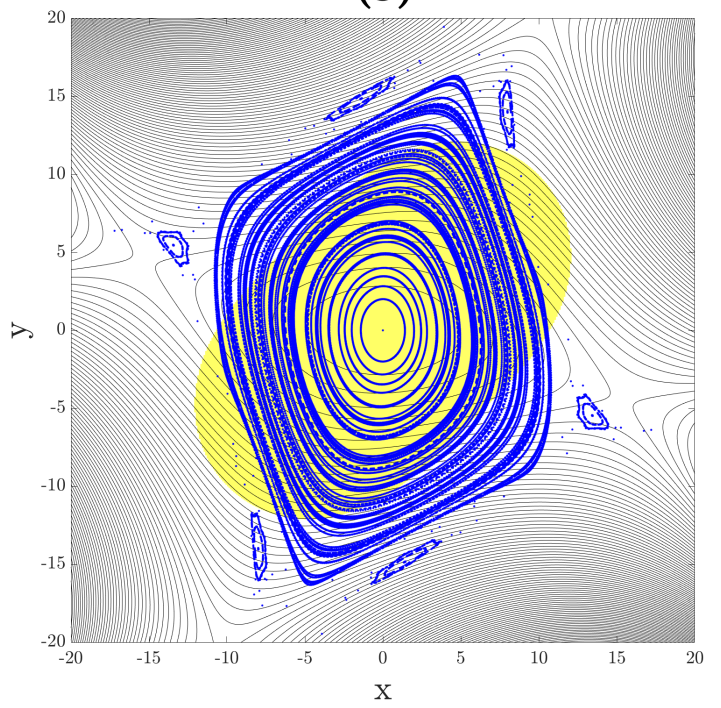

FIG. 6. (a) Instantaneous streamlines and Okubo-Weiss elliptic region (yellow) for the universal NavierStokes solution (36) with $\alpha(t) \equiv 0.005$ at time $t=0$. Other time slices are similar. (b) KAM curves (blue) of the same velocity field.

solution (36), both the Okubo-Weiss criterion and the instantaneous streamlines correctly predict the presence of a coherent vortex around the origin. At the same time, they fail to predict the correct shape of the vortex, and completely miss six smaller vortices surrounding the large vortex in Fig. 6(b).

\section{v. CONCLUSIONS}

We have derived an explicit form for all spatially polynomial, universal, planar Navier-Stokes flows up to arbitrary order. We then used examples of such solutions to test the ability of the instantaneous streamlines and of the Okubo-Weiss criterion, the $2 \mathrm{D}$ version of the $Q$-criterion, to detect coherent material vortices and stretching regions in unsteady flows.

Specifically, using the main result of this paper, we have derived two chaotically mixing Navier-Stokes flows whose analysis via instantaneous streamlines and by the Okubo-Weiss criterion suggests a lack of stretching due to the presence of a coherent vortex. Likewise, we have constructed two exact Navier-Stokes flows that have a bounded coherent Lagrangian vortex around 
the origin despite the hyperbolic flow structure suggested by instantaneous streamlines and the Okubo-Weiss criterion. Finally, we have constructed a Navier-Stokes solution whose trajectories form a coherent vortex near the origin. While the Okubo-Weiss criterion and the instantaneous streamlines do signal a nearby vortex in this example, they fail to render the correct shape of the vortex and miss additional smaller vortices in its neighborhood.

Using the 2D unsteady solutions, we have given an explicit family of unsteady polynomial solutions to the 3D Navier-Stokes equation. The first two coordinates of these 3D velocity fields agree with our planar polynomial solutions, while their third coordinate is simply a uniform, constant velocity component. When applied to these extended unsteady solutions, the $Q_{-}^{-}, \Delta_{-}, \lambda_{2}$ - and $\lambda_{c i}$-criteria give the same incorrect flow classification results as the Okubo-Weiss criterion does in our two-dimensional examples.

The exact solutions derived in this paper can be used as basic unsteady benchmarks for coherent structure detection criteria and numerical schemes. They also provide a wealth of bounded, dynamically consistent flow patterns away from boundaries. For instance, the specific two-dimensional velocity field examples we have derived can be viewed as models of coherent structures, such as eddies and fronts, in oceanic flows away from the coastlines.

\section{ACKNOWLEDGMENTS}

We would like to acknowledge useful conversations with Mattia Serra on the subject of this paper. This work was partially supported by the Turbulent Superstructures Program of the German National Science Foundation (DFG).

\section{REFERENCES}

${ }^{1}$ A. Majda, "Vorticity and the mathematical theory of incompressible fluid flow," Commun. Pure. Appl. Math. 39, S187-S220 (1986).

${ }^{2}$ A. J. Majda and A. L. Bertozzi, Vorticity and incompressible flow (Cambridge University Press, 2002).

${ }^{3}$ A. D. D. Craik and W. O. Criminale, "Evolution of wavelike disturbances in shear flows: a class of exact solutions of the Navier-Stokes equations," in Proceedings of the Royal Society of 
Explicit Unsteady Navier-Stokes Solutions and their Analysis via Local Vortex Criteria

London A: Mathematical, Physical and Engineering Sciences, 1830 (The Royal Society, 1986) pp. 13-26.

${ }^{4}$ R. Berker, "Intégration des équations du mouvement d'un fluide visqueux incompressible," Handbuch der Physik VIII/2, 1-384 (1963).

${ }^{5}$ C. Y. Wang, "Exact solutions of the unsteady Navier-Stokes equations," Appl. Mech. Rev. 42, S269-S282 (1989).

${ }^{6}$ C. Y. Wang, "Exact solutions of the Navier-Stokes equations-the generalized Beltrami flows, review and extension," Acta Mech. 81, 69-74 (1990).

${ }^{7}$ C. Y. Wang, "Exact solutions of the steady-state Navier-Stokes equations," Ann. Rev. Fluid Mech. 23, 159-177 (1991).

${ }^{8}$ P. G. Drazin and N. Riley, The Navier-Stokes equations: a classification of flows and exact solutions, 334 (Cambridge University Press, 2006).

${ }^{9}$ A. E. Perry and M. Chong, "A series-expansion study of the Navier-Stokes equations with applications to three-dimensional separation patterns," Journal of fluid mechanics 173, 207-223 (1986).

${ }^{10}$ T. R. Bewley and B. Protas, "Skin friction and pressure: the footprints of turbulence," Physica D: Nonlinear Phenomena 196, 28-44 (2004).

${ }^{11}$ K. Bajer and H. K. Moffatt, "On a class of steady confined stokes flows with chaotic streamlines," Journal of Fluid Mechanics 212, 337-363 (1990).

${ }^{12}$ J. C. R. Hunt, A. Wray, and P. Moin, "Eddies, streams, and convergence zones in turbulent flows," Center for turbulence research report CTR-S88 1, 193-208 (1988).

${ }^{13}$ M. S. Chong, A. E. Perry, and B. J. Cantwell, “A general classification of three-dimensional flow fields," Phys Fluids A-Fluid 2, 765-777 (1990).

${ }^{14}$ J. Jeong and F. Hussain, "On the identification of a vortex," J. Fluid Mech. 285, 69-94 (1995).

${ }^{15}$ P. Chakraborty, S. Balachandar, and R. J. Adrian, "On the relationships between local vortex identification schemes," J. Fluid Mech. 535, 189-214 (2005).

${ }^{16}$ G. E. Andrews, R. Askey, and R. Roy, Special functions (Cambridge University Press, 2000).

${ }^{17}$ A. Okubo, "Horizontal dispersion of floatable particles in the vicinity of velocity singularities such as convergences," in Deep-Sea Res. (Elsevier, 1970) pp. 445-454.

${ }^{18} \mathrm{~J}$. Weiss, "The dynamics of enstrophy transfer in two-dimensional hydrodynamics," Physica D 48, 273-294 (1991).

${ }^{19}$ B. Epps, "Review of vortex identification methods," in 55th AIAA Aerospace Sciences Meeting, 
Explicit Unsteady Navier-Stokes Solutions and their Analysis via Local Vortex Criteria

https://arc.aiaa.org/doi/pdf/10.2514/6.2017-0989.

${ }^{20}$ G. Rousseaux, S. Seifer, V. Steinberg, and A. Wiebel, “On the lamb vector and the hydrodynamic charge," Experiments in fluids 42, 291-299 (2007).

${ }^{21}$ M. Belevich, "Non-relativistic abstract continuum mechanics and its possible physical interpretations," Journal of Physics A: Mathematical and Theoretical 41, 045401 (2008).

${ }^{22} \mathrm{H}$. Marmanis, “Analogy between the navier-stokes equations and maxwell's equations: Application to turbulence," Physics of Fluids 10, 1428-1437 (1998).

${ }^{23}$ S. Sridhar, "Turbulent transport of a tracer: An electromagnetic formulation," Phys. Rev. E 58, 522-525 (1998).

${ }^{24}$ G. Haller, “An objective definition of a vortex,” J. Fluid Mech. 525, 1-26 (2005).

${ }^{25}$ G. Haller, "Lagrangian coherent structures,” Annual Review of Fluid Mechanics 47, 137-162 (2015).

${ }^{26}$ H. J. Lugt, "The dilemma of defining a vortex," in Recent developments in theoretical and experimental fluid mechanics (Springer, 1979) pp. 309-321.

${ }^{27}$ I. A. Sadarjoen and F. H. Post, "Detection, quantification, and tracking of vortices using streamline geometry," Computers \& Graphics 24, 333-341 (2000).

${ }^{28}$ S. K. Robinson, "Coherent motions in the turbulent boundary layer," Annual review of fluid mechanics 23, 601-639 (1991).

${ }^{29}$ V. I. Arnold, Mathematical methods of classical mechanics (Springer, 1989).

${ }^{30}$ V. Arnold, "Sur la topologie des écoulements stationnaires des fluides parfaits," C. R. Acad. Sci. 261, 17 (1965).

${ }^{31}$ J. T. Ault, K. K. Chen, and H. A. Stone, "Downstream decay of fully developed dean flow," Journal of Fluid Mechanics 777, 219-244 (2015).

${ }^{32}$ J.-M. Baey and X. J. Carton, "Piecewise-constant vortices in a two-layer shallow-water flow," in IUTAM Symposium on Advances in Mathematical Modelling of Atmosphere and Ocean Dynamics (Springer, 2001) pp. 87-92.

${ }^{33}$ X.-d. Bai, W. Zhang, Q.-h. Fang, Y. Wang, J.-h. Zheng, and A.-x. Guo, “The visualization of turbulent coherent structure in open channel flow, Journal of Hydrodynamics 31, 266-273 (2019).

${ }^{34}$ G. I. Bell and L. J. Pratt, "Eddy-jet interaction theorems for piecewise constant potential vorticity flows," Dynamics of atmospheres and oceans 20, 285-314 (1994).

${ }^{35}$ S. Childress, "New solutions of the kinematic dynamo problem," J. Math. Phys. 11, 3063-3076 (1970). 
Explicit Unsteady Navier-Stokes Solutions and their Analysis via Local Vortex Criteria

${ }^{36}$ M. F. A. Couette, études sur le frottement des liquides, Ph.D. thesis, Gauthier-Villars (1890).

${ }^{37}$ K. Dayal and R. D. James, "Design of viscometers corresponding to a universal molecular simulation method," Journal of Fluid Mechanics 691, 461-486 (2012).

${ }^{38}$ K. Dayal and R. D. James, "Nonequilibrium molecular dynamics for bulk materials and nanostructures,” J. Mech. Phy. Solids. 58, 145-163 (2010).

${ }^{39}$ T. Dombre, U. Frisch, J. M. Greene, M. Hénon, A. Mehr, and A. M. Soward, "Chaotic streamlines in the abc flows," J. Fluid Mech. 167, 353-391 (1986).

${ }^{40}$ T. Dumitrică and R. D. James, “Objective molecular dynamics,” J. Mech. Phy. Solids. 55, 22062236 (2007).

${ }^{41}$ S. Dupont and Y. Brunet, “Coherent structures in canopy edge flow: a large-eddy simulation study," Journal of Fluid Mechanics 630, 93-128 (2009).

${ }^{42}$ G. Haller and T. Sapsis, "Lagrangian coherent structures and the smallest finite-time lyapunov exponent,' Chaos: An Interdisciplinary Journal of Nonlinear Science 21, 023115 (2011).

${ }^{43} \mathrm{~K}$. Hiemenz, Die Grenzschicht an einem in den gleichförmigen Flüssigkeitsstrom eingetauchten geraden Kreiszylinder, Ph.D. thesis, Universität Göttingen (1911).

${ }^{44}$ B. L. Hua and P. Klein, “An exact criterion for the stirring properties of nearly two-dimensional turbulence," Physica D 113, 98-110 (1998).

${ }^{45}$ B. L. Hua, J. C. McWilliams, and P. Klein, "Lagrangian accelerations in geostrophic turbulence," Journal of Fluid Mechanics 366, 87-108 (1998).

${ }^{46}$ T. von Kármán, “Über laminare und turbulente reibung," ZAMM-Journal of Applied Mathematics and Mechanics/Zeitschrift für Angewandte Mathematik und Mechanik 1, 233-252 (1921).

${ }^{47}$ V. Kolář, "Vortex identification: New requirements and limitations," International journal of heat and fluid flow 28, 638-652 (2007).

${ }^{48}$ J.-P. Mollicone, F. Battista, P. Gualtieri, and C. M. Casciola, "Turbulence dynamics in separated flows: The generalised kolmogorov equation for inhomogeneous anisotropic conditions," Journal of Fluid Mechanics 841, 1012-1039 (2018).

${ }^{49}$ C. W. Oseen, Über Wirbelbewegung in einer reibenden Flüssigkeit (Almqvist \& Wiksells, 1911).

${ }^{50}$ A. E. Overman II, "Steady-state solutions of the euler equations in two dimensions ii. local analysis of limiting v-states," SIAM Journal on Applied Mathematics 46, 765-800 (1986).

${ }^{51}$ A. E. Perry, “A series expansion study of the navier-stokes equations," NASA STI/Recon Technical Report N 85, 18306 (1984).

${ }^{52} \mathrm{~J}$. L. Poiseuille, Recherches expérimentales sur le mouvement des liquides dans les tubes de 
Explicit Unsteady Navier-Stokes Solutions and their Analysis via Local Vortex Criteria

très-petits diamètres (Imprimerie Royale, 1844).

${ }^{53} \mathrm{G}$. G. Stokes, On the effect of the internal friction of fluids on the motion of pendulums (Pitt Press, 1851).

${ }^{54} \mathrm{C}$. Truesdell and K. R. Rajagopal, An introduction to the mechanics of fluids (Springer Science \& Business Media, 2010).

${ }^{55}$ Á. Viúdez, "Elliptic and hyperbolic interior solutions of piecewise-constant potential vorticity geophysical vortices," Journal of Fluid Mechanics 696, 301-318 (2012).

${ }^{56}$ D. Zwillinger, Handbook of differential equations (Gulf Professional Publishing, 1998).

${ }^{57}$ Y. Zhang, X. Qiu, F. Chen, K. Liu, X. Dong, and C. Liu, “A selected review of vortex identification methods with applications," Journal of Hydrodynamics 30, 767-779 (2018). 